\title{
471351 - ARTERIAL SPIN LABELLING FUNCTIONAL MRI - A NEW MODALITY FOR SUCCESSFULLY CAPTURING CEREBRAL BLOOD FLOW CHANGES ASSOCIATED WITH TONIC PAIN
}

\author{
Collin Clarke, M.D. ${ }^{1}$, Sue Ganapathy, M.D. ${ }^{1}$, Keith St. Lawrence, Ph.D ${ }^{2}$, Daron \\ Owen, M.Sc ${ }^{2}$ \\ 1. Anesthesia, UWO, London, ON, Canada \\ 2. Medical Imaging, Lawson Health Research Institute, London, ON, Canada
}

Introduction: Functional magnetic resonance (fMRI) imaging has become the dominant tool for mapping neuronal activity in both research and clinical studies. This is due to its safety, non-invasiveness and its generation of images with excellent temporal and special resolution. The majority of fMRI studies use blood oxygenation level dependent (BOLD) contrast as a marker for neural activation. BOLD has limitations including considerable variability in the BOLD signal between subjects and low-frequency 'drifts' in the MR signal with time, thus BOLD studies are generally restricted to activation paradigms that can be performed in a cyclical on/off pattern., These factors make BOLD a poor modality for mapping cerebral changes associated with tonic pain paradigms. Recent data suggest that a new technique in imaging called arterial spin labeling (ASL) shows stable noise characteristics over the entire frequency spectrum and less inter-subject variability, potentially making it ideal for studying low-frequency events in brain function such as changes in tonic pain. The purpose of this study is to determine if ASL can be used successfully to follow cerebral changes associated with tonic pain.

Methods: Following approval from the University Research Ethics Board. The study was conducted on a 3 Tesla scanner. Prior to imaging, a catheter (size $25 \mathrm{G}$ ) was inserted into the flexor muscle in the forearm of the non-dominate hand. An intramuscular infusion of hypertonic (5\%) saline was connected. The functional study consists of two parts: a 10minute resting period, followed by a 20-minute pain period during which hypertonic (5\%) saline is continuously infused. Images of cerebral blood flow are acquired throughout the functional study using our ASL technique. During the pain period, the infusion rate was gradually titrated increased to a pain rating of $7 / 10$ was attained. Results: Suprathreshold t-statistics, corrected for multiple comparisons (FDR, $\mathrm{p}<0.05$ ), were performed for the entire 20 minutes and for the first 5 minutes. In both analyses, significant increases in CBF were seen in the Insula, Pallidum, Putamen, Thalamus, and S2. Activations seen in the 5 minute period but not the complete duration included anterior and middle cingulate cortex. Larger activation in the first 5 minutes indicate that this early period is responsible for a large part of the activation over the entire 20 minutes. The lack of cingulate activation in the total duration may reflect a decrease in the affective component of the pain over time.

Discussion: The larger activation in the first 5 minutes indicates that the initial pain response may be responsible for a large part of the activation over the entire 20 minutes. The lack of cingulate activation in the later time period may reflect a decrease in the affective component of the pain over time. This study demonstrates that ASL is able to detect the CBF changes associated clinically-relevent pain stimulus and thus may be a 
useful tool for the study of intervention effects on chronic pain References: 1) Pain. 2007 [Epub ahead of print] 2) Proc. Natl. Acad. Sci. U.S.A 1998;95:1834-1839. 3) Magn Reson Med. 2003;50:99-106 4) Magn Reson Med. 2003;49:796-802. 